\title{
Radioactive decay of GRB-SNe at late-times
}

\author{
Kuntal Misra $^{1}$ and A. S. Fruchter ${ }^{2}$ \\ ${ }^{1}$ Aryabhatta Research Institute of observational sciencES, \\ Manora Peak, Nainital India 263002 \\ email: kuntal@aries.res.in \\ ${ }^{2}$ Space Telescope Science Institute, \\ 3700 San Martin Drive, Baltimore, MD USA 21210 \\ email: fruchter@stsci.edu
}

\begin{abstract}
We present the late-time Hubble Space Telescope observations of two Gamma Ray Burst (GRB) associated supernovae (SNe), GRB 030329/SN 2003dh and XRF 060218/SN 2006aj. Using the multi-color data up to $\sim 320$ days after the burst, we constrain the latetime decay nature of these SNe. The decay rates of SN 2003dh are steeper than SN 2006aj. A comparison with two other GRB SNe, GRB 980425/SN 1998bw and the SN associated with XRF 020903, shows that the decay rates of SN 2003dh are similar to XRF 020903 and those of SN 2006aj are similar to SN 1998bw. The late-time decay rates are steeper than the ${ }^{56} \mathrm{Co} \rightarrow{ }^{56} \mathrm{Fe}$ radioactive decay rate indicating that there is some leakage of gamma-rays. We also compare the late-time decay rates of nine type Ic SNe, including the SNe of long GRBs, Ic broad lined and normal Ics. The decay rates of the SNe sample show a remarkable similarity in $I$ band at late-times with a scatter of $\sim 10 \%$.
\end{abstract}

Keywords. gamma ray bursts, (stars:) supernovae: general, (stars:) supernovae: individual (SN 2003dh, SN 2006aj)

\section{Introduction}

Long duration Gamma Ray Bursts (GRBs) are thought to be associated to a special type of stripped envelope supernovae ( $\mathrm{SNe}$ ) that have lost most of their outer $\mathrm{H}$ and He envelope (type Ic). A few SNe out of the type Ic's show broad spectral lines with very high expansion velocities $(\sim 30,000 \mathrm{~km} / \mathrm{s})$ and are known as 'broad lined' type Ic SNe. Till date all SNe associated with GRBs and X-Ray Flashes (XRFs) which have been spectroscopically typed have been found to be type Ic SNe. Because of the energetic nature of explosion, the SNe associated to GRBs are often termed as 'hypernovae'. While there is accumulating evidence that nearly all low redshift GRBs have an underlying broad lined type Ic SN, not all energetic broad lined type Ic's show a GRB association.

The first GRB to be associated with a SN, GRB 980425, was detected by the Gamma Ray Burst Monitor onboard the BeppoSAX satellite and further spectroscopic observations confirmed that the new optical transient in the error box of GRB 980425 was indeed a very luminous SN (named SN 1998bw) and was classified as a type Ic SN (Galama et al. 1998, Iwamoto et al. 1998). However, it was with the discovery of GRB 030329/SN 2003dh that the association of GRBs with SNe became incontrovertible and provided the first unambiguous evidence of a SN association with a long duration GRB. Since then about a dozen more SNe have been spectroscopically confirmed to have a GRB association with spectral features which are clearly of broad lined type Ic SNe (see Cano et al. 2016 for a review). 
The SNe light curves are powered by the radioactive decay of ${ }^{56} \mathrm{Ni} \rightarrow{ }^{56} \mathrm{Co} \rightarrow{ }^{56} \mathrm{Fe}$. The peak bolometric luminosity gives an estimate of ${ }^{56} \mathrm{Ni}$ synthesized in the explosion (Arnett 1982). The discovery of new GRB-SN associations and broad lined type Ic SNe is revealing a large diversity in terms of the ${ }^{56} \mathrm{Ni}$ production, ejecta mass and the explosion energy. The diversity in the ${ }^{56} \mathrm{Ni}$ produced comes in mainly because type Ic's show a lot of variability in the peak luminosity. Typically the broad lined type Ic without a GRB are found to be less luminous with smaller explosion energy and ejecta mass as compared to hypernovae associated to GRBs. The physical parameters for most of the GRB associated SNe are estimated from the information obtained using the early time light curve and spectrum. It is only in a few cases, like SN 1998bw where late-time data is available, that an estimation of physical parameters is possible (Patat et al. 2001) using the nebular phase data. In the case of GRBs it is not easy to construct late-time light curves of associated SNe because GRBs are typically distant objects and hence lack detailed study at late-times due to limited data.

In this paper, we examine the SNe associated with two long duration GRBs - SN 2003dh/GRB 030329 and SN 2006aj/XRF 060218 which are unique because of the availability of the Hubble Space Telescope (HST) data allowing us to constrain the decay nature of the late-time light curve of the SNe. Due to the proximity and nature of these GRB/XRF-SNe, the early afterglow and SN light curves of both SN 2003dh/GRB 030329 $(\mathrm{z}=0.1685)$ and SN 2006aj/XRF $060218(\mathrm{z}=0.033)$ have been studied by several authors in detail (Hjorth et al. 2003; Matheson et al. 2003; Stanek et al. 2003; Deng et al. 2005; Resmi et al. 2005; Cobb et al. 2006; Mazzali et al. 2006; Mirabal et al. 2006; Modjaz et al. 2006; Pian et al. 2006; Sollerman et al. 2006). The subset of GRB-SNe are compared with a sample of broad lined type Ic and normal type Ic supernovae which have published late-time light curves.

\section{Data Reduction}

HST observed the afterglow of GRB 030329 and the associated supernova SN 2003dh in $F 606 \mathrm{~W}$ and $F 814 \mathrm{~W}$ colors from $\sim 17$ to 772 days after the burst. The last epoch $H S T$ observations at $\sim 772$ and 422 days after the burst in $F 606 \mathrm{~W}$ and $F 814 \mathrm{~W}$ colors respectively are used to estimate the host galaxy flux. At late-times the contribution of the afterglow to the total flux becomes negligible and the supernova flux dominates. We therefore use the HST data beyond $\sim 72$ days after the burst when the contribution from the afterglow becomes insignificant. The late-time observations of XRF 060218/SN 2006aj spanning $\sim 150-260$ days after the burst were carried out at four epochs in four colors - F435W, F555W, F625W and F814W. We do not have SN 2006aj host galaxy images free from supernova flux to estimate the host galaxy flux.

The HST data were processed using the multidrizzle routine within the stsdas package in IRAF (Fruchter \& Hook 2002). We used a pixfrac of 0.8 and a scale of 0.03333 to obtain the final drizzled image. Thus, the final images have a pixel size of $0.033 \mathrm{arcsec} / \mathrm{pixel}$. The residual image in GRB 030329 is created by subtracting the last epoch image from the early epochs and is shown in Figure 1. In the subtracted image we accurately know the position of the optical transient and estimate the supernova flux in a fixed aperture. In the case of SN 2006aj/XRF 060218 subtracting the early epochs from the last epoch gives a clear detection of the optical transient and a very accurate position as shown in Figure 1. In the case of SN 2006aj the supernova flux is measured in a small aperture; the total flux thus contains the contribution of the host galaxy within this aperture. For both the sources, the corresponding AB magnitudes are estimated using the ACS zero points. 

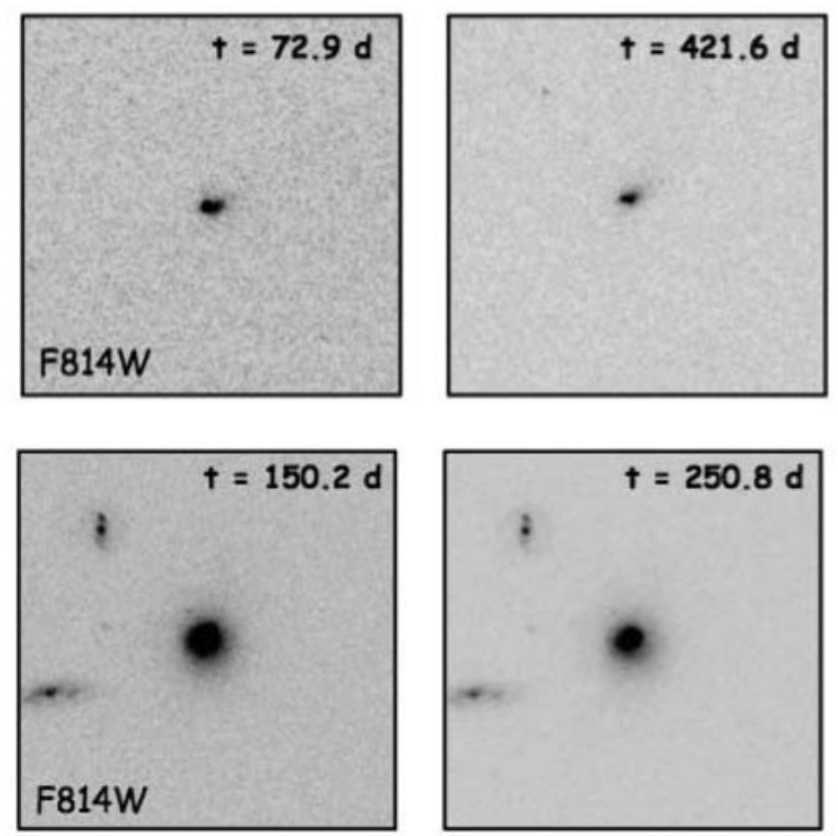

Residual
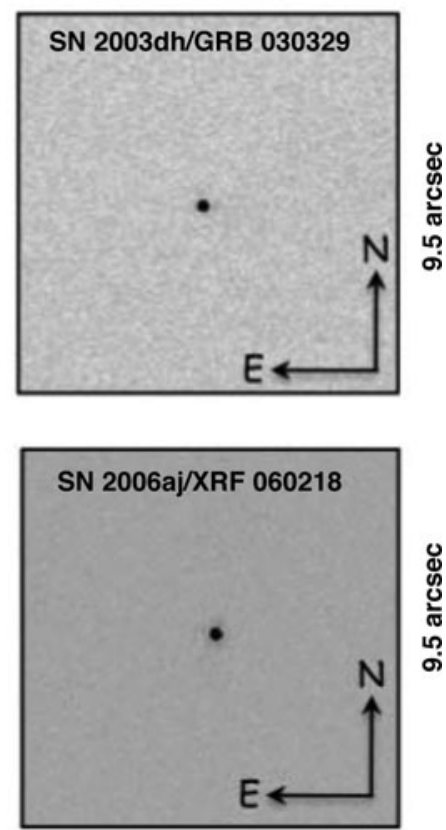

Figure 1. HST ACS imaging of SN 2003dh/GRB 03029 and SN 2006aj/XRF 060218 in F814W filter at two epochs as shown in the figure. The residual image is created by subtracting the last epoch image from early epochs.

\section{Analysis and Preliminary Results}

The unique data set of both SN 2003dh and SN 2006aj allows us to study the latetime behaviour of the light curve. The flux estimates of SN 2003dh are free of any host contamination as discussed in Section 2. To these fluxes we fit an exponential $(F(t)=$ $A e^{-\alpha t}$ where $A$ is the normalization and $\alpha$ is the decay rate) and estimate the late-time decay rates. In the case of SN 2006aj since we do not have host galaxy flux measurements we add a constant $C$ to the above mentioned exponential form in order to account for the host galaxy flux within the aperture. The decay rates for SN 2003dh and SN 2006aj as obtained from the exponential fits and the corresponding errors are listed in Table 1 and shown in Figure 2.

We also compare the late-time light curves of SN 2003dh and SN 2006aj to two other GRB associated SNe - GRB 980425/SN 1998bw (Patat et al. 2001) and the supernova associated with XRF 020903 (Bersier et al. 2006). The decay rates of these two supernovae are taken from the literature and are listed in Table 2 for a comparison with our results on SN 2003dh and SN 2006aj. The apparent magnitudes published in the literature are converted to $\mathrm{AB}$ magnitudes for a direct comparison with $\mathrm{AB}$ magnitudes derived from the HST observations. The absolute magnitudes have been derived from the observed photometry using a cosmology of $H_{0}=70 \mathrm{~km} \mathrm{~s}^{-1} \mathrm{Mpc}^{-1}, \Omega_{m}=0.27$ and $\Omega_{\Lambda}=0.73$. For each SNe we plot the absolute magnitudes in Figure 2 corrected for the luminosity distance and total (Galactic+Host) extinction. We see that the decay rates of SN 2006aj are remarkably similar to those of SN $1998 \mathrm{bw}$ at late-times except for the $B$ band. On the other hand we see that the decay rates are steeper in SN 2003dh and they are similar to the decay seen in the supernova associated to XRF 020903 (Table 2). In Figure 2 we have also shown the ${ }^{56} \mathrm{Co} \rightarrow{ }^{56} \mathrm{Fe}$ radioactive decay $\left(0.0098 \mathrm{mag}\right.$ day $\left.{ }^{-1}\right)$ in the case of complete 
Table 1. Late-time decay rates of GRB associated supernovae

\begin{tabular}{ccccc}
\hline Object & $B$ & $V$ & $R$ & $I$ \\
\hline GRB 030329/SN 2003dh & - & $0.0218 \pm 0.001$ & - & $0.0189 \pm 0.001$ \\
XRF 060218/SN 2006aj & $0.0114 \pm 0.004$ & $0.0186 \pm 0.001$ & $0.0166 \pm 0.0006$ & $0.0170 \pm 0.0008$
\end{tabular}

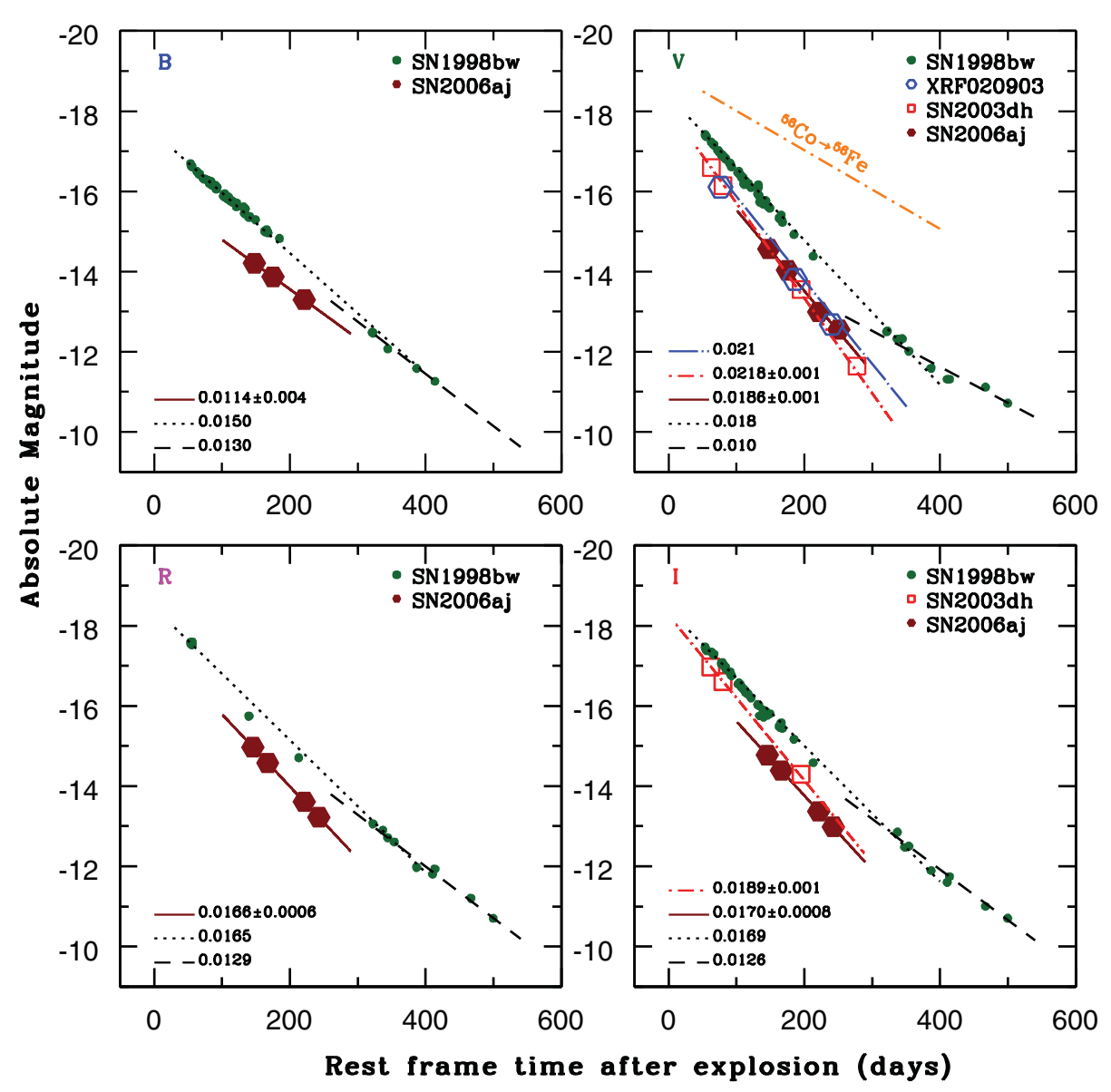

Figure 2. A comparison of multi-color absolute magnitudes of SN 1998bw (Galama et al. 1998; McKenzie et al. 1999; Sollerman et al. 2000; Patat et al. 2001), the supernova associated with XRF 020903 (Bersier et al. 2006), SN 2003dh (this work) and SN 2006aj (this work). The dotted and dashed lines represent the decay rates of SN 1998bw in the ranges 54-312 and 321-500 days respectively. The dash-dotted line corresponds to the ${ }^{56} \mathrm{Co} \rightarrow{ }^{56} \mathrm{Fe}$ decay rate, as expected if there was no leakage of $\gamma$-rays.

$\gamma$-ray trapping. We see that the decay rates of all the supernovae shown in Figure 2 are steeper than the ${ }^{56} \mathrm{Co} \rightarrow{ }^{56} \mathrm{Fe}$ decay rates indicating that there is some leakage of $\gamma$-rays. For a comparison, in SN 1998bw we also show the data in the time range of 324-504 days after the burst (Figure 2$)$. At these late phases $(t>300$ days), the multi-band light curves of SN 1998bw show a clear flattening and the light curve settles onto a ${ }^{56} \mathrm{Co} \rightarrow{ }^{56} \mathrm{Fe}$ decay phase. 
Table 2. A comparison of type Ic SNe late-time decay rates in the rest frame

\begin{tabular}{|c|c|c|c|c|c|}
\hline Object & $B$ & $V$ & $R$ & $I$ & Reference \\
\hline & & GRB-SNe & & & \\
\hline \multicolumn{6}{|c|}{ GRB $980425 / \mathrm{SN} 1998 \mathrm{bw}$} \\
\hline 41-309 days & $0.015 \pm 0.001$ & & & & Patat et al. 2001 \\
\hline $41-323$ days & & $0.018 \pm 0.0006$ & $0.0165 \pm 0.0019$ & $0.0169 \pm 0.001$ & \\
\hline $308-400$ days & $0.013 \pm 0.0008$ & & & & \\
\hline $308-486$ days & & $0.0101 \pm 0.0008$ & $0.0129 \pm 0.0006$ & $0.0126 \pm 0.0008$ & \\
\hline \multicolumn{6}{|c|}{ XRF $020903 / \mathrm{SN}$} \\
\hline $76-240$ days & & 0.021 & & & Bersier et al. 2006 \\
\hline \multicolumn{6}{|c|}{ GRB $030329 / \mathrm{SN} 2003 \mathrm{dh}$} \\
\hline $63-273$ days & & $0.0218 \pm 0.001$ & & & This work \\
\hline 63-195 days & & & & $0.0189 \pm 0.001$ & \\
\hline \multicolumn{6}{|c|}{ XRF $060218 / \mathrm{SN} 2006 \mathrm{aj}$} \\
\hline 148-221 days & $0.0114 \pm 0.004$ & & & & This work \\
\hline $148-250$ days & & $0.0186 \pm 0.001$ & & & \\
\hline $146-253$ days & & & $0.0166 \pm 0.0006$ & $0.0170 \pm 0.0008$ & \\
\hline
\end{tabular}

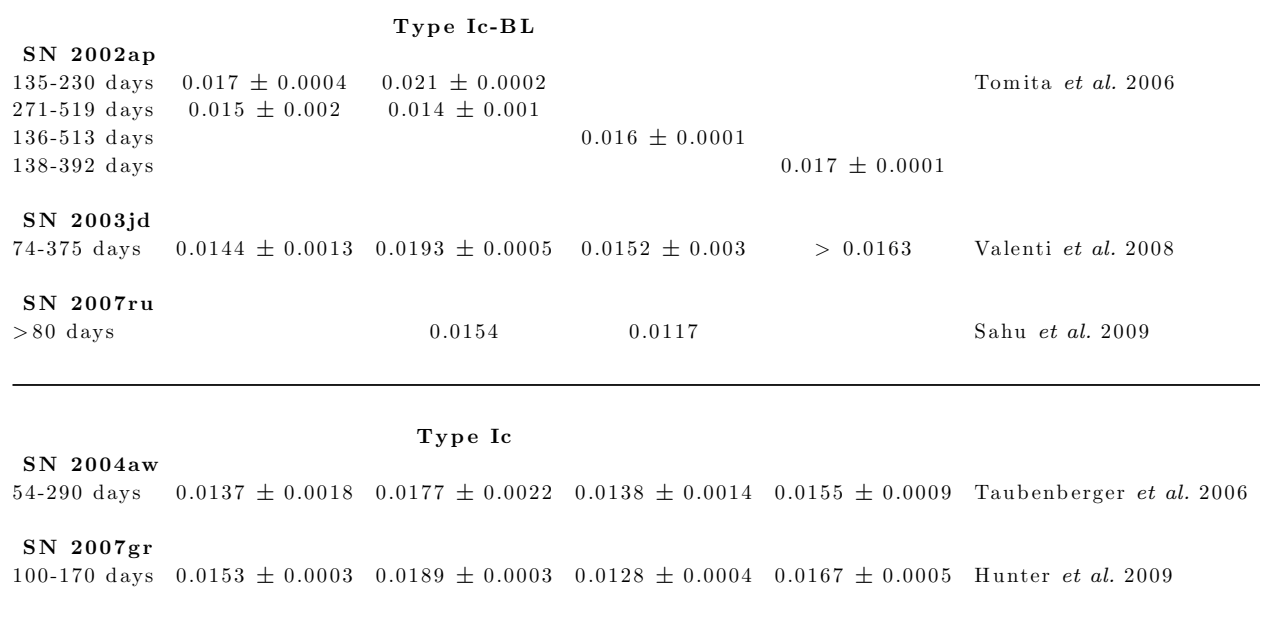

We further increase our comparison sample by including the broad lined type Ic SNe that are not associated with any GRB/XRF and the normal type Ic SNe which have light curves published in the literature. We found three broad lined type Ic SNe (SNe 2002ap, 2003jd and 2007ru) without any GRB/XRF association and two normal type Ic SNe (SNe 2004aw and 2007gr) with published data extending out to reasonably late-times (nearly 170-500 days). Altogether we have a sample of nine type Ic SNe. In Table 2 we list the rest frame decay rates of the SNe in our sample in different colors at various time ranges. A comparison of the late-time decay rates of the SNe in our sample shows that the decay rates are fairly similar in the $I$ band with a scatter of $\sim 10 \%$. These decay rates are steeper than ${ }^{56} \mathrm{Co} \rightarrow{ }^{56} \mathrm{Fe}$ radioactive decay indicating that the $\gamma$-ray leakage seen in all type Ic SNe is standard. 


\section{Conclusions}

We present the late-time HST ACS multi-color observations of two GRB associated SNe - GRB 030329/SN 2003dh and XRF 060218/SN 2006aj. Both these GRB associated supernovae are unique because of the availability of late-time data which allows us to constrain the decay behaviour at late-times. The SNe associated with GRB 980425/SN 1998bw and XRF 020903 are others for which late-time observations exist. The decay rates of SNe 2006aj and 1998bw are remarkably similar at late-times while those of SN 2003dh are steeper and similar to the SN associated with XRF 020903. In a larger sample of type Ic SNe including the broad lined type Ic with and without a GRB association and normal type Ic SNe, we find that the reported late-time decay rates (Table 2) are fairly similar in the $I$ band with a scatter of $\sim 10 \%$. It is also to be noted that the decay rates are steeper than ${ }^{56} \mathrm{Co} \rightarrow{ }^{56} \mathrm{Fe}$ radioactive decay indicating that the $\gamma$-ray leakage seen in all type Ic SNe is standard.

\section{Acknowledgements}

We thank the organizers of IAU Symposium 331 "SN 1987A, 30 years later - cosmic rays and nuclei from supernovae and their aftermaths" to provide an opportunity to present our work. KM acknowledges the financial support received from IAU to attend the symposium.

\section{References}

Arnett, W. D., 1982, ApJ, 253, 785

Bersier, D. et al., 2006, ApJ, 643, 284

Cano, Z. et al., 2016, arXiv: 1604.03549

Cobb, B. E. et al., 2006, ApJ, 645, L113

Deng, J. et al., 2005, ApJ, 624, 898

Fruchter, A. S. \& Hook, R. N., 2002, PASP, 114, 144

Galama, T. J. et al., 1998, Nature, 395, 670

Hjorth, J. et al., 2003, Nature, 423, 847

Hunter, D. J. et al., 2009, A\& $A, 508,371$

Iwamoto, K. et al., 1998, ApJ, 534, 660

Matheson, T. et al., 2003, ApJ, 599, 394

Mazzali, P. A. et al., 2006, Nature, 442, 1018

McKenzie, E. H. et al., 1999, PASP, 111, 964

Mirabal, N. et al., 2006, ApJ, 643, L99

Modjaz, M. et al., 2006, ApJ, 645, L21

Patat, F. et al., 2001, ApJ, 555, 900

Pian, E. et al., 2006, Nature, 442, 1011

Resmi, L. et al., 2005, A\&A A, 440, 477

Sahu, D. K. et al., 2009, ApJ, 697, 676

Sollerman, J. et al., 2006, A\&AA, 454, 503

Sollerman, J. et al., 2000, ApJ, 537, L127

Stanek, K. Z. et al., 2003, ApJ, 591, L17

Taubenberger, S. et al., 2006, MNRAS, 371, 1459

Tomita, H. et al., 2006, ApJ, 644, 400

Valenti, S. et al., 2008, MNRAS, 383, 1485 